\title{
Building on a genetic framework: Can we personalize the timing of surgical repair for patients with heritable thoracic aortic disease?
}

Ashley Dawson, MD, ${ }^{\mathrm{a}}$ and Scott A. LeMaire, $\mathrm{MD}^{\mathrm{a}, \mathrm{b}, \mathrm{c}, \mathrm{d}}$

Feature Editor's Introduction-Cardiovascular genetics is a nascent but promising arena to improve and personalize care. Recent advances in technology, efficiency, and accessibility of genetic sequencing has increased the pace and quantity of scientific discovery. However, information alone does not translate to clinical management. The interpretation of genetic variation requires an integration with individual clinical information, including family history. In hereditary thoracic aortopathies, the relative lack of symptoms in concert with the life-threatening sequelae of dissection or rupture render early identification and screening paramount. As genetic sequencing technology continues apace, the potential for "incidental" discovery remains. Commercial availability of with direct-to-consumer genetic testing kits, wherein health care providers' roles may be minimized, is another area for concern.

In this edition of the Journal, Dawson and LeMaire highlight the work of the Aortopathy Working Group in the context of the Clinical Genome Resource. Through curation, synthesis of evidence, and classification of the strength of gene-disease relationships in aortopathies, the group has built a framework on which to construct patient-specific recommendations. Dawson and LeMaire also provide a brief review of aortopathies in general, and specific recommendations for individual heritable aortopathies. This work supplements the current guidelines on the management of thoracic aortic diseases, providing more granular detail on when and whom to refer for testing, and when to intervene.

Dawn S. Hui, MD, and Richard Lee, MD, MBA

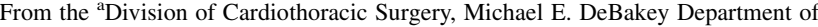
Surgery, ${ }^{\mathrm{c}}$ Cardiovascular Research Institute, and ${ }^{\mathrm{d}}$ Department of Molecular Physiology and Biophysics, Baylor College of Medicine, Houston, Tex; and ${ }^{\mathrm{b}}$ Division of Cardiovascular Surgery, Texas Heart Institute, Houston, Tex.

Dr Dawson is supported by a fellowship award through the University of KentuckyBaylor College of Medicine Aortopathy Research Center within the American Heart Association Strategically Focused Vascular Disease Research Network (18SFRN33960114). Dr LeMaire is supported in part by the Jimmy and Roberta Howell Professorship in Cardiovascular Surgery at Baylor College of Medicine.

Received for publication Nov 11, 2019; revisions received Jan 28, 2020; accepted for publication Jan 30, 2020; available ahead of print June 17, 2020.

Address for reprints: Scott A. LeMaire, MD, Michael E. DeBakey Department of Surgery, Baylor College of Medicine, One Baylor Plaza, BCM 390, Houston, TX 77030 (E-mail: slemaire@bcm.edu).

J Thorac Cardiovasc Surg 2020;160:901-5 $0022-5223 / \$ 36.00$

Copyright (c) 2020 Published by Elsevier Inc. on behalf of The American Association for Thoracic Surgery

https://doi.org/10.1016/j.jtcvs.2020.01.121
}

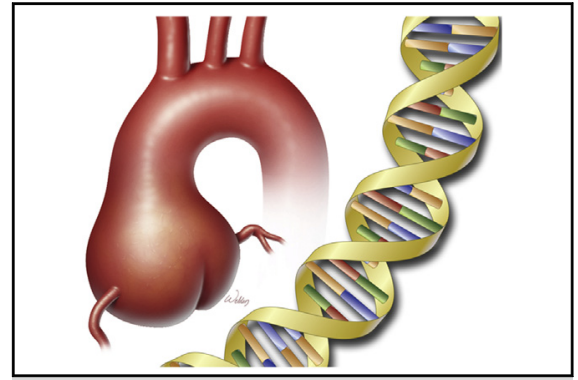

Aortic aneurysms in heritable aortic disease typically involve the aortic root.

CENTRAL MESSAGE

The expansion of genetic research combined with the rigorous requirements for identifying clinically valid genes has important implications for the surgical management of heritable aortic disease.

This Invited Expert Opinion provides a perspective on the following paper: J Am Coll Cardiol. 2018;72:605-615. https://doi.org/10.1016/j.jacc.2018. 04.089 .

See Commentary on page 906.

Thoracic aortic aneurysms are life-threatening and often asymptomatic. Identifying patients who are at risk and initiating an early screening regimen can improve the timing of operative intervention and reduce the incidence of fatal complications such as dissection and rupture. In many cases, thoracic aortic aneurysms and dissections (TAAD) are genetically triggered. Current research and clinical observations indicate that heritable aortic disease is linked to autosomal dominant single-gene disorders that predominantly involve the aortic root and ascending aorta initially. ${ }^{1,2}$

Advancements in the accessibility and efficiency of genetic sequencing have led to an increasingly long list of candidate genes potentially associated with TAAD. Because the screening recommendations and timing of intervention depend on the disorder, restricting diagnostic panels to genes that are strongly associated with clinically 
relevant aortic disease is important for meaningful counseling and surveillance and for minimizing false positives. The problem of excessive potential gene-disease relationships-many with conflicting evidence-is not unique to aortic disease. To address this, the Clinical Genome Resource (ClinGen) has developed a curation process that synthesizes clinical and experimental evidence to determine the strength of a gene-disease relationship. ${ }^{3}$ Within the Cardiovascular Disease Working Group in ClinGen, an Aortopathy Working Group was assembled, including international experts in clinical and molecular genetics, to evaluate genes by using the rigorous curation methodology and analytical framework developed by the ClinGen Gene $\mathrm{Cu}$ ration Working Group. ${ }^{4}$ Using this methodology, the Aortopathy Working Group made up of Renard and colleagues ${ }^{4}$ sought to classify the strength of evidence for genes that potentially predispose patients to hereditable TAAD (HTAAD). For a gene to show a "strong" association with a disease, a role for that gene in the disease must be shown in at least 2 independent studies and in numerous unrelated probands, and the evidence must be supported by compelling gene-level experimental data. For a gene to show a "definitive" association with a disease, these findings must be upheld over time (at least 3 years).

The Aortopathy Working Group evaluated 53 candidate genes selected from the literature and clinical aortopathy gene test panels. Probands were examined for gene pathogenicity on the basis of the variant type and protein domain involved. A standardized, semiquantitative assessment of the available evidence revealed 11 genes that had a "definitive" or "strong" association with HTAAD. These genes were considered "clinically actionable" with implications for gene-specific management in screening, surveillance, and surgical management. Four genes were classified as having "limited evidence," and 23 genes were classified as having "no clinical evidence" for an association with HTAAD, with the supporting literature based solely on experimental data. Several of these genes with "limited" or no "clinical evidence," including $C B S$, COL5A1, COL5A2, MED12, and PLOD1, are currently included on multiple aortopathy comprehensive gene testing panels. Because these genes have not been shown to contribute to clinically relevant aortic disease, removing them from comprehensive genetic screening may minimize unnecessary stress to patients and reduce the need for surveillance. In addition, 7 genes were identified as "uncertain"; that is, having insufficient evidence to currently assign a classification. These genes are expected to be recurated as new data become available, and ongoing screening of these genes in the interim is recommended. The progressive expansion of genetic research will continue to provide new targets and evidence for genes associated with HTAAD, resulting in the aortopathy-curated gene list becoming a living tool that evolves with the literature.
Currently, surgical intervention is indicated when the risk of rupture or dissection is substantial. In patients with hereditable thoracic aortic aneurysm, this risk is not directly associated with aneurysm diameter and varies among disorders. ${ }^{1,5,6}$ Determining which genes are clinically associated with HTAAD allows clinicians to study each variant's progression and to form more specific clinical histories. A better understanding of how heritable aortic disease manifests will provide a framework for developing gene- and mutation-specific recommendations for the timing of repair.

\section{SYNDROMIC VERSUS NONSYNDROMIC THORACIC AORTIC DISEASE}

Heritable thoracic aortic disease may be categorized as syndromic or nonsyndromic. The syndromic form presents within a disease such as Marfan syndrome (MFS) in which multiple organ systems are affected by the mutation. Syndromic disease phenotypes can be used for diagnosis; however, because several syndromic phenotypes overlap, confirmation with genetic testing is useful.

In nonsyndromic disease, the effect of the genetic mutation appears to be limited to the cardiovascular system. In these cases, understanding the pathogenic variant can help to determine a probable disease course and identify other family members at risk.

\section{Syndromic Disorders}

MFS. MFS is characterized by manifestations in the eyes, skeletal system, and cardiovascular system; these features contribute to the overall phenotype used for diagnosis. In 2010, new diagnostic criteria for Marfan syndrome were released, known as the revised Ghent nosology, and have given more weight to aortic aneurysm or dissection in the diagnosis of MFS. ${ }^{7}$ The relationship between the extracellular matrix glycoprotein fibrillin-1 (encoded by FBN1) and MFS has been well established in the literature ${ }^{2,8}$; as expected, $F B N 1$ was classified as "definitive" by Renard and colleagues. ${ }^{4} F B N 1$ mutations have been shown to affect both structural and cell signaling processes, leading to aortic root aneurysms with characteristic morphology (Figure 1) and mitral valve prolapse. ${ }^{9,10}$ Notably, $90 \%$ of patients with MFS require an aortic operation during their lifetime. Because cardiothoracic and vascular manifestations are largely responsible for morbidity and mortality in MFS patients, operative timing is an important factor in a patient's disease management. Performing elective root replacement before aortic dissection occurs has been shown to improve life expectancy. ${ }^{11,12}$ For patients with FBNI mutations, aortic root replacement is recommended if the external diameter reaches $5.0 \mathrm{~cm}$. An even lower threshold for diameter is recommended for patients with high-risk characteristics such as rapid aortic expansion, a family history of aortic dissection or rupture, or severe systemic features. $^{13,14}$ 


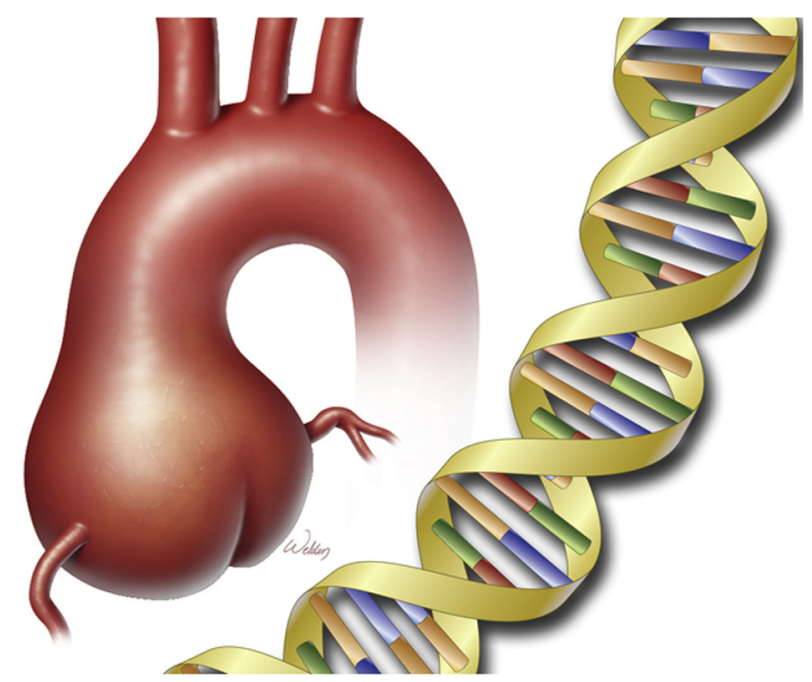

FIGURE 1. Aortic aneurysms in heritable aortic disease typically involve the aortic root. The illustration shows the characteristic morphology of aortic root aneurysms in patients with Marfan syndrome. Used with permission of Baylor College of Medicine.

Loeys-Dietz syndrome and other transforming growth factor $\beta$ vasculopathies. The phenotype associated with transforming growth factor (TGF)- $\beta$ vasculopathies ranges from isolated TAAD to the severe systemic features present in patients with Loeys-Dietz syndrome (LDS). ${ }^{15-17}$ LDS is characterized by some clinical features that are similar to those in MFS, including skeletal and cutaneous manifestations, and was not recognized as a separate disease until 2005. ${ }^{16}$ Genes with mutations known to cause TGF- $\beta$ vasculopathies include TGFBR1, TGFBR2, TGFB2, and SMAD3. ${ }^{15,17}$ These genes were noted to be "definitive" in their association with HTAAD. ${ }^{4}$ TGFB3 has also been implicated in $\mathrm{HTAAD}^{18}$; however, this gene is currently classified as "uncertain" with potential for reclassification. ${ }^{4}$

In patients with TGF- $\beta$ vasculopathies, cardiovascular manifestations such as arterial tortuosity and aortic aneurysm are more severe, with progression to aortic dissection at an earlier age and at smaller aortic diameters than in MFS. ${ }^{19}$ The risk of vascular complications is increased in patients with systemic features (ie, arterial tortuosity, small body surface area, hypertelorism, and wide scars), rapid expansion of the aneurysm, or a family history of early aortic dissection or rupture. ${ }^{15}$ Notably, one-third of patients with LDS experience an aortic event (eg, dissection, surgery, or death resulting from dissection or rupture) before 19 years of age. ${ }^{17}$ Women with TGFBR2 mutations, small body surface area, and syndromic features appear to be at particularly high risk. ${ }^{15}$ Therefore, for patients with mutations involving TGFBRl, $T G F B R 2$, or $S M A D 3$, ascending aortic repair is recommended if the aortic diameter reaches $4 \mathrm{~cm}$ in patients with risk factors or $4.5 \mathrm{~cm}$ in patients without risk factors. $^{13}$
Shprintzen-Goldberg syndrome. Shprintzen-Goldberg syndrome is associated with a genetic mutation in the $S K I$ gene that can result in systemic features similar to those in MFS and LDS. ${ }^{20}$ Unlike MFS or LDS, aortic disease is not the defining feature in Shprintzen-Goldberg syndrome, and cardiovascular abnormalities are generally less severe. Although the evidence for $S K I$ as a direct causative gene for HTAAD was classified as "moderate," it remains beneficial on aortopathy gene panels to distinguish ShprintzenGoldberg syndrome from other syndromes with a higher risk of aortic complications.

Vascular Ehlers-Danlos syndrome. Vascular EhlersDanlos syndrome (ie, Ehlers-Danlos type IV) is caused by pathologic variants in the collagen gene COL3A1. This disease is characterized by fragile vascular tissues, and aortic dissection occurs at smaller aortic diameters than in the other syndromic disorders. ${ }^{21}$ Because a strong association has been consistently observed between pathogenic variants of COL3Al and acute aortic dissection, COL3A1 was classified as "definitive" in its association with HTAAD. ${ }^{4}$ To minimize the risk of dissection, repair is recommended in the appropriate patients when aortic diameter reaches $4.5 \mathrm{~cm} .{ }^{13}$

\section{Nonsyndromic Disorders}

Patients who present with isolated cardiovascular disease without systemic features are classified as nonsyndromic. At least $20 \%$ of patients with non-syndromic TAAD have been shown to have a strong family history; these cases are termed familial thoracic aortic aneurysms (FTAA). ${ }^{13}$

In the absence of syndromic features, genetic testing is recommended for patients who present with TAAD at a young age (younger than age 50 years) or with a positive family history. ${ }^{22,23}$ The Netherlands National Working Group on Bicuspid Aortic Valve and Thoracic Aortic Aneurysms has also recommended testing patients between ages of 50 and 60 years who present with TAAD without a history of hypertension. ${ }^{23}$ Genetic testing in patients with nonsyndromic HTAAD involves using panels that include a large set of known causative genes.

The American College of Cardiology Foundation/American Heart Association guidelines for the management of thoracic aortic aneurysm specify that mutations in $F B N 1, T G F B R 1$, TGFBR2, COL3A1, ACTA2, or MYH11 prompt further aortic imaging in first-degree relatives of patients with TAAD. ${ }^{14}$ Those genes, as well as SMAD3, TFGB2, MYLK, LOX, and $P R K G 1$ are classified as "strong or definitive" in their relationship to HTAAD by Renard and colleagues. ${ }^{4}$ An ideal aortopathy gene panel would include these "strong or definitive" genes and minimize variants of uncertain significance.

Screening beyond first-degree relatives may also be indicated. A recent systematic review by Mariscalco and colleagues $^{24}$ revealed that nonsyndromic thoracic aortic disease affected more than $30 \%$ of first-degree and $20 \%$ 
of second-degree relatives. However, increased screening for FTAA highlights the importance of using only rigorously validated genes when making clinical decisions based on genetic variants. Testing positive for a variant of uncertain significance may result in family members undergoing costly imaging for a disease they may not be at risk for developing. Alternatively, testing negative for a variant may provide false reassurance. Although genetic screening has become less expensive, the cost of testing does not account for imaging and surveillance studies after a positive result. Cost analysis of aortopathy screening was not performed in any of the studies included in the review by Mariscalco and colleagues, ${ }^{24}$ indicating an area for future study.

Because of the increased likelihood of aortic dissection at smaller aortic diameters in patients with FTAA, the optimal recommended timing of surgery in these patients is generally when aortic aneurysm diameter reaches $4.5 \mathrm{~cm} .{ }^{13,14}$ In patients who have a strong family history without a genetic diagnosis, repair is recommended at $5.0 \mathrm{~cm} .{ }^{13}$ Recommendations for the timing of repair in patients with FTAA may become more specific as the likelihood of dissection associated with each gene is better understood. Mariscalco and colleagues $^{24}$ showed that aortic dissection occurred at aortic diameters smaller than $4.5 \mathrm{~cm}$ in only some of the genes studied, including FBN1, FOXE2, MYLK, PRKG1, SMAD3, TGFB2, TGFBR1, and TGFBR2. Other genes associated with FTAA, such as ACTA2, LOX, and MYH11, were associated with dissection at diameters larger than $5.5 \mathrm{~cm}$. These findings suggest that risk stratification could be used to determine the timing of surgical repair for patients with nonsyndromic HTAAD. The standardized reporting of measurement location and methodology for each genetic variant will also be helpful in consolidating the results of different studies to make definitive recommendations.

\section{FURTHER CONSIDERATIONS IN THE TIMING OF REPAIR}

In addition to stratifying risk based on genetic diagnosis, other patient factors must be considered when counseling patients on the timing of repair. Measuring the indexed aortic diameter along with the true aortic diameter may be helpful, particularly in women or patients with smaller stature. Some authors have advocated using maximal aortic aneurysm cross-sectional area divided by the patient's height as an approach to adjusting for body size, with a recommendation to consider surgery when this value exceeds $10 .{ }^{14}$ Concerning features such as a family history of early disease progression or an increased rate of expansion (more than $0.5 \mathrm{~cm} /$ year) indicate the need for earlier repair. ${ }^{14}$ Increased vessel tortuosity has also been shown to be associated with an increased risk of adverse outcomes. ${ }^{25}$

The risk of aortic dissection is markedly increased during pregnancy in patients with HTAAD because of changes in hemodynamic parameters, as well as in the aortic media. ${ }^{26}$
Therefore, preconception counseling and imaging of the entire aorta are recommended. Elective aortic root repair is generally recommended in patients with MFS when aortic aneurysm size exceeds $4.5 \mathrm{~cm}$, or in patients with LDS when aortic aneurysm size exceeds $4.0 \mathrm{~cm} .{ }^{13}$ In patients with FTAA, aortic root repair is generally recommended at an aneurysm size of $5.0 \mathrm{~cm}$ or larger. ${ }^{13}$ During pregnancy, patients remain at risk for aortic events distal to the ascending aorta, and frequent evaluation is indicated. ${ }^{15}$

Bicuspid aortic valve (BAV) is also associated with nonsyndromic TAAD. Compared with patients who have triscuspid aortic valve, a history of BAV alone has not been shown to be a risk factor for dissection at smaller aneurysm sizes, and repair is recommended at $5.5 \mathrm{~cm}^{13,27}$ Screening the firstdegree family members of patients with BAV for both valvular abnormalities and ascending aortic aneurysm is recommended. ${ }^{14}$ If a patient is undergoing aortic valve replacement, concomitant prophylactic replacement of the ascending aorta is considered if the aortic diameter is larger than $4.5 \mathrm{~cm}{ }^{13,27}$

\section{CONCLUSIONS AND FUTURE DIRECTIONS}

Genetic sequencing has significantly increased in power and availability and has immense potential for improving the diagnosis and management of HTAAD. However, many of the gene-disease relationships identified through genetic sequencing have been questionable, resulting in potentially unnecessary stress and surveillance. The curation methodology used by the Aortopathy Working Group ${ }^{4}$ provides a living framework for evaluating and re-evaluating genes with a potential relationship to HTAAD. As more data are acquired, new genes with "definitive" or "strong relationships" to HTAAD will continue to be identified. With this ongoing expansion of data from researchers studying heritable aortic disease, standardizing reporting methods is critical. Renard and colleagues ${ }^{4}$ have provided a table of minimal clinical and genetic data that should be reported. The consistent reporting of phenotypic features and cardiovascular disease presentation will optimize future gene curation.

The increased ability to provide patients with a specific genetic diagnosis that can be confidently linked to aortic disease has important implications. A definitive genetic diagnosis itself may improve outcomes by prompting patients and physicians to have a high index of suspicion for aortic complications. For example, patients with known MFS have been shown to have a shorter length of time from the onset of symptoms to the diagnosis of acute aortic dissection. ${ }^{28}$ As more data are collected, increasingly personalized recommendations for patients with HTAAD are possible. In addition to the variation in the timing of surgical intervention described above, knowledge of the expected clinical course for a given pathogenic variant allows for more precise risk stratification in patients undergoing the medical management of hereditary aortic disease. This may help guide the choice of antihypertension or 
antihyperlipidemia medications. Understanding an individual's risk of developing aortic dissection or aneurysm rupture may also be helpful when weighing the risks and benefits of starting a medication that is potentially detrimental to the aortic wall. For example, fluoroquinolone use has been clinically associated with an increased risk of aortic aneurysm and dissection. ${ }^{29,30}$ These findings are supported by recent work from our lab showing in a mouse model of sporadic aortic aneurysm and dissection that the administration of ciprofloxacin increased rates of aortic dissection and rupture. Identifying genes associated with HTAAD that also show increased susceptibility to fluoroquinolones may be helpful in selecting an antibiotic regimen in patients with HTAAD. ${ }^{31}$

Genetic research continues to expand and has important implications for the treatment of HTAAD. The gene curation effort by the Aortopathy Working Group ${ }^{4}$ has established a list of "clinically valid" genes in HTAAD, with the potential for adding new genes as data become available. Limiting the genes used in the clinical setting to only those that are well validated ensures that evidencebased information is used when counseling patients with a specific pathogenic variant.

\section{Conflict of Interest Statement}

The authors reported no conflicts of interest.

The Journal policy requires editors and reviewers to disclose conflicts of interest and to decline handling or reviewing manuscripts for which they may have a conflict of interest. The editors and reviewers of this article have no conflicts of interest.

The authors thank Scott A. Weldon, MA, CMI, FAMI, for creating the illustration and Nicole Stancel, PhD, ELS(D), at the Texas Heart Institute, for providing editorial support.

\section{References}

1. Isselbacher EM, Lino Cardenas CL, Lindsay ME. Hereditary influence in thoracic aortic aneurysm and dissection. Circulation. 2016;133:2516-28.

2. Lindsay ME, Dietz HC. Lessons on the pathogenesis of aneurysm from heritable conditions. Nature. 2011;473:308-16

3. Strande NT, Riggs ER, Buchanan AH, Ceyhan-Birsoy O, DiStefano M, Dwight SS, et al. Evaluating the clinical validity of gene-disease associations: an evidence-based framework developed by the clinical genome resource. Am J Hum Genet. 2017;100:895-906.

4. Renard M, Francis C, Ghosh R, Scott AF, Witmer PD, Ades LC, et al. Clinical validity of genes for heritable thoracic aortic aneurysm and dissection. $J$ Am Coll Cardiol. 2018;72:605-15.

5. Coady MA, Rizzo JA, Goldstein LJ, Elefteriades JA. Natural history, pathogenesis, and etiology of thoracic aortic aneurysms and dissections. Cardiol Clin. 1999; 17:615-35.vii.

6. Davies RR, Goldstein LJ, Coady MA, Tittle SL, Rizzo JA, Kopf GS, et al. Yearly rupture or dissection rates for thoracic aortic aneurysms: simple prediction based on size. Ann Thorac Surg. 2002;73:17-27.

7. Loeys BL, Dietz HC, Braverman AC, Callewaert BL, De Backer J, Devereux RB, et al. The revised Ghent nosology for the Marfan syndrome. J Med Genet. 2010;47:476-85.

8. Dietz HC, Cutting GR, Pyeritz RE, Maslen CL, Sakai LY, Corson GM, et al. Marfan syndrome caused by a recurrent de novo missense mutation in the fibrillin gene. Nature. 1991;352:337-9.
9. Neptune ER, Frischmeyer PA, Arking DE, Myers L, Bunton TE, Gayraud B, et al. Dysregulation of TGF-beta activation contributes to pathogenesis in Marfan syndrome. Nat Genet. 2003;33:407-11.

10. Ramachandra CJ, Mehta A, Guo KW, Wong P, Tan JL, Shim W. Molecular pathogenesis of Marfan syndrome. Int J Cardiol. 2015;187:585-91.

11. Pyeritz RE. Recent progress in understanding the natural and clinical histories of the Marfan syndrome. Trends Cardiovasc Med. 2016;26:423-8.

12. Silverman DI, Burton KJ, Gray J, Bosner MS, Kouchoukos NT, Roman MJ, et al Life expectancy in the Marfan syndrome. Am J Cardiol. 1995;75:157-60.

13. Ouzounian M, LeMaire SA. How can genetic diagnosis inform the decision of when to operate? J Vis Surg. 2018;4:68.

14. Hiratzka LF, Bakris GL, Beckman JA, Bersin RM, Carr VF, Casey DE Jr, et al 2010 ACCF/AHA/AATS/ACR/ASA/SCA/SCAI/SIR/STS/SVM guidelines for the diagnosis and management of patients with thoracic aortic disease. A report of the American College of Cardiology Foundation/American Heart Association task force on practice guidelines, American Association for Thoracic Surgery, American College of Radiology, American Stroke Association, Society of Cardiovascular Anesthesiologists, Society for Cardiovascular Angiography and Interventions, Society of Interventional Radiology, Society of Thoracic Surgeons, and Society for Vascular Medicine. J Am Coll Cardiol. 2010;55:e27-129.

15. Jondeau G, Ropers J, Regalado E, Braverman A, Evangelista A, Teixedo G, et al. International registry of patients carrying TGFBRI or TGFBR2 mutations: results of the MAC (Montalcino Aortic Consortium). Circ Cardiovasc Genet. 2016;9:548-58.

16. Loeys BL, Chen J, Neptune ER, Judge DP, Podowski M, Holm T, et al. A syndrome of altered cardiovascular, craniofacial, neurocognitive and skeletal development caused by mutations in TGFBR1 or TGFBR2. Nat Genet. 2005;37:275-81.

17. Loeys BL, Schwarze U, Holm T, Callewaert BL, Thomas GH, Pannu H, et al. Aneurysm syndromes caused by mutations in the TGF-beta receptor. $N$ Engl $J$ Med. 2006;355:788-98.

18. Bertoli-Avella AM, Gillis E, Morisaki H, Verhagen JMA, de Graaf BM, van de Beek G, et al. Mutations in a TGF-beta ligand, TGFB3, cause syndromic aortic aneurysms and dissections. J Am Coll Cardiol. 2015;65:1324-36.

19. Williams JA, Loeys BL, Nwakanma LU, Dietz HC, Spevak PJ, Patel ND, et al. Early surgical experience with Loeys-Dietz: a new syndrome of aggressive thoracic aortic aneurysm disease. Ann Thorac Surg. 2007;83:S757-63.

20. Doyle AJ, Doyle JJ, Bessling SL, Maragh S, Lindsay ME, Schepers D, et al. Mutations in the TGF-beta repressor SKI cause Shprintzen-Goldberg syndrome with aortic aneurysm. Nat Genet. 2012;44:1249-54.

21. Pepin M, Schwarze U, Superti-Furga A, Byers PH. Clinical and genetic features of Ehlers-Danlos syndrome type IV, the vascular type. N Engl J Med. 2000;342:673-80

22. Milewicz DM, Regalado E. GeneReviews ${ }^{\circledR}$ [Internet]. In: Adam MP, Ardinger HH, Pagon RA, Wallace SE, Bean LJH, Stephens K, et al., eds. Heritable Thoracic Aortic Disease Overview. Seattle, WA: University of Washington, Seattle; 2003.

23. Verhagen JMA, Kempers M, Cozijnsen L, Bouma BJ, Duijnhouwer AL, Post JG, et al. Expert consensus recommendations on the cardiogenetic care for patients with thoracic aortic disease and their first-degree relatives. Int J Cardiol. 2018; 258:243-8.

24. Mariscalco G, Debiec R, Elefteriades JA, Samani NJ, Murphy GJ. Systematic review of studies that have evaluated screening tests in relatives of patients affected by nonsyndromic thoracic aortic disease. J Am Heart Assoc. 2018;7:e009302.

25. Morris SA, Orbach DB, Geva T, Singh MN, Gauvreau K, Lacro RV. Increased vertebral artery tortuosity index is associated with adverse outcomes in children and young adults with connective tissue disorders. Circulation. 2011;124:388-96.

26. Wanga S, Silversides C, Dore A, de Waard V, Mulder B. Pregnancy and thoracic aortic disease: managing the risks. Can J Cardiol. 2016;32:78-85.

27. Hiratzka LF, Creager MA, Isselbacher EM, Svensson LG, Nishimura RA Bonow RO, et al. Surgery for aortic dilatation in patients with bicuspid aortic valves: a statement of clarification from the American College of Cardiology/ American Heart Association task force on clinical practice guidelines. J Thorac Cardiovasc Surg. 2016;151:959-66.

28. de Beaufort HWL, Trimarchi S, Korach A, Di Eusanio M, Gilon D, Montgomery DG, et al. Aortic dissection in patients with Marfan syndrome based on the IRAD data. Ann Cardiothorac Surg. 2017;6:633-41.

29. Daneman N, Lu H, Redelmeier DA. Fluoroquinolones and collagen associated severe adverse events: a longitudinal cohort study. BMJ Open. 2015;5:e10077.

30. Lee CC, Lee MT, Chen YS, Lee SH, Chen YS, Chen SC, et al. Risk of aortic dissection and aortic aneurysm in patients taking oral fluoroquinolone. JAMA Intern Med. 2015;175:1839-47.

31. LeMaire SA, Zhang L, Luo W, Ren P, Azares AR, Wang Y, et al. Effect of ciprofloxacin on susceptibility to aortic dissection and rupture in mice. JAMA Surg. 2018;153:e181804. 